Relations industrielles

Industrial Relations

\title{
Y a-t-il un âge pour travailler ? par François Hubault (dir.), Toulouse : Octarès Éditions, 2012, 132 pages. ISBN : EAN9782366300079 et ISSN : 1778-5030.
}

\section{Siham Abouaissa}

Volume 69, numéro 3, été 2014

URI : https://id.erudit.org/iderudit/1026763ar

DOI : https://doi.org/10.7202/1026763ar

Aller au sommaire du numéro

Éditeur(s)

Département des relations industrielles de l’Université Laval

ISSN

0034-379X (imprimé)

1703-8138 (numérique)

Découvrir la revue

Citer ce compte rendu

Abouaissa, S. (2014). Compte rendu de [Y a-t-il un âge pour travailler ? par François Hubault (dir.), Toulouse : Octarès Éditions, 2012, 132 pages. ISBN : EAN9782366300079 et ISSN : 1778-5030.] Relations industrielles / Industrial Relations, 69(3), 648-651. https://doi.org/10.7202/1026763ar

Tous droits réservés (C Département des relations industrielles de l’Université Laval, 2014
Ce document est protégé par la loi sur le droit d'auteur. L'utilisation des services d'Érudit (y compris la reproduction) est assujettie à sa politique d'utilisation que vous pouvez consulter en ligne.

https://apropos.erudit.org/fr/usagers/politique-dutilisation/ 
en plus de tâches sont, par conséquence directe, confiées à des collaborateurs extérieurs, c'est-à-dire des pigistes. Les collectifs comme le Terrier d'Hégésippe peuvent dès lors facilement faire office de rédaction externalisée, d'agences de production de contenu travaillant comme sous-traitant » (p. 307).

En conclusion, Un salariat au-delà du salariat? analyse deux figures emblématiques des emplois et des parcours contemporains, en mettant en évidence non seulement la précarité de ces travailleurs, mais aussi leurs aspirations à travailler autrement. Elles ont en commun une position mitoyenne sur un continuum opposant le salariat typique et l'indépendance pure, puisqu'il s'agit de travailleurs bénéficiant d'une forte autonomie professionnelle, à qui le droit du travail français a étendu le pouvoir protecteur du salariat. Comme le font toutefois remarquer les codirectrices de l'ouvrage, seuls les intermittents bénéficient d'un régime particulier d'indemnisation du chômage et c'est donc surtout à partir de leur cas qu'est posée la question de la sécurisation des trajectoires professionnelles. Les journalistes pigistes, quant à eux, cotisent au régime général dont ils ne peuvent que rarement bénéficier.

Au-delà du cas français, des deux groupes étudiés et de la conjoncture particulière dans laquelle la recherche a été conduite, la lecture de cet ouvrage présente un intérêt certain pour le lecteur intéressé par les défis contemporains de la protection sociale en contexte d'éclatement de la norme d'emploi. Il ne s'agit plus, comme dans les dispositifs classiques d'assurance, de protéger les travailleurs contre un risque exceptionnel, mais de les supporter collectivement dans un parcours intrinsèquement discontinu. Parce qu'elle analyse à partir de multiples perspectives, les transformations historiques de dispositifs ayant tenté d'atteindre cet objectif, cette contribution permet une réflexion concrète sur l'objectif de sécurisation des trajectoires professionnelles, en soulevant ces deux questions fondamentales: qu'est-ce que le travail et qu'est-ce qui doit donner droit à la protection?

Sur une note plus critique, les chapitres présentent un degré d'approfondissement inégal. Certains constituent des résumés d'éléments déjà bien documentés de la condition des créateurs pigistes. D'autres, au contraire, pour être bien compris, requièrent de connaître minimalement le régime d'indemnisation du chômage des intermittents du spectacle en France, ce qui pourra constituer un obstacle pour le lecteur novice

\section{Martine D'Amours}

Université Laval

\section{Y a-t-il un âge pour travailler ?}

par François Hubault (dir.), Toulouse : Octarès Éditions, 2012, 132 pages.

ISBN : EAN9782366300079 et ISSN : 1778-5030.

"Y a-t-il un âge pour travailler?", un ouvrage collectif coordonné par François Hubault, réunit les contributions d'un séminaire organisé par le Département d'ergonomie et écologie humaine de l'Université Paris 1. Cet ouvrage propose " une mise en perspective des enjeux soulevés par le thème du vieillissement au travail ». Les contributions proposées sont celles d'ergonomes, mais aussi d'un inspecteur de travail, d'un médecin du travail, d'un psychologue et d'un directeur des ressources humaines.

La question que pose le titre de l'ouvrage est d'actualité et elle permet, somme toute, plusieurs éléments de réponse selon le positionnement des acteurs impliqués dans le milieu du travail. Par ailleurs, on constate, depuis quelque temps, la recrudescence des thèmes " âge », " travail », " expérience », etc. dans des ouvrages essentiellement composés de contributions d'ergonomes traitant de la question du vieillissement en emploi. En effet, l'ergonomie fait partie des 
disciplines les plus actives dans la recherche sur ce sujet, ce qui peut expliquer le grand nombre de travaux publiés.

Cet ouvrage, comme ceux qui l'ont précédé, porte sur la question de l'âge au travail à travers une série de thèmes habituellement traités, notamment la dimension différentielle de l'âge, les déficits fonctionnels et les stratégies de régulation des travailleurs afin de composer avec de tels déficits, la pénibilité et ses conséquences, de même que le manque de prise en considération de ces réalités par les organisations, qui pourtant gagneraient à gérer les âges sans discrimination. En bref, il y a là plusieurs thèmes qu'un spécialiste du vieillissement en emploi ou un lecteur averti pourrait retrouver dans bon nombre de travaux précédents (notamment l'ouvrage de Molinié et al., 2012). La question que nous nous sommes donc posée est la suivante : quelle nouveauté cet ouvrage apporte-t-il au débat? Afin d'y répondre, nous avons effectué une recension des différentes contributions présentes dans l'ouvrage.

Tout d'abord, François Hubault présente, de manière générale, les différents thèmes développés par les différents auteurs. II met en exergue la question de l'âge dans l'organisation ainsi que toutes les " problématiques » qui en découlent, à savoir l'âgisme prépondérant dans certaines entreprises, la pénibilité, etc. Par ailleurs, Hubault apporte des débuts de réponse à ces différents questionnements, en tentant de mieux appréhender les effets de la relation « âgetravail » dans les organisations.

Catherine Delgoulet poursuit la réflexion amorcée par Hubault en abordant la relation " âge-travail » dans la perspective de I'amélioration de la démarche des ergonomes dans leurs interventions. Pour ce faire, elle décrit d'abord le contexte démographique et socio-économique actuel ainsi que les réalités du vieillissement au travail (notamment du point de vue des représen- tations sociales du vieillissement). Après avoir évoqué les différentes situations de travail (individuel ou collectif, formation, régulations suite au changement de l'organisation du travail, etc.), Delgoulet revient ensuite sur les enjeux de la relation « âgetravail » que les ergonomes ont à relever, cela afin d'améliorer le traitement de cette problématique. L'auteure prône alors une approche "diachronique » qui permettrait de prendre en compte la diversité et la pluralité des situations existantes dans le marché de l'emploi.

Yves Roquelaure traite de la question de I'âge au travail du point de vue de la physiopathologie'. Cette discipline permet, selon l'auteur, d'apporter " une vision renouvelée et intégrée " des relations entre I'âge, I'ancienneté et l'intensité de l'exposition professionnelle. En effet, les TMS-MS (troubles musculo-squelettiques des membres supérieurs) sont des maux courants dans le milieu du travail et constituent la première cause de maladies professionnelles en Europe. Les expériences ont montré que ces troubles ont tendance à s'accumuler avec l'âge. Or, I'individualisation du travail ainsi que le manque de régulations collectives et de soutien social conduisent parfois à l'exclusion les travailleurs souffrant de ce handicap, plus particulièrement des plus âgés d'entre eux. Roquelaure parvient à la conclusion que, malgré les efforts consentis de prévention de ces maux, il y a des risques que les TMS-MS continuent de se développer. Selon lui, l'effort de prévention actuel ne prend pas en compte plusieurs aspects dégénératifs, ce qui a pour effet que ces troubles s'accumulent au fur et à mesure du temps et deviennent, ainsi, chroniques. Une prévention plus poussée et menée en amont (prévention de l'apparition chez les futurs travailleurs exposés) et en aval (amélioration de la qualité de

1 La physiopathologie est l'étude des troubles de fonctionnement de l'organisme ou de ses parties, au cours des maladies. 
vie des travailleurs atteints) serait plus à même d'apporter des résultats.

Dominique Cau-Bareille s'intéresse au vieillissement dans le secteur de l'enseignement dans les maternelles de France. Son objectif est de comprendre les exigences du travail de ces enseignantes, notamment au moment de leur fin de carrière. Ainsi, I'auteure met en exergue les différentes formes de pénibilité (physique, mentale et psychique) ainsi que les transformations du métier (intensification du travail, manque de temps, etc.) auxquelles doivent faire face ces salariées. Cau-Bareille souligne notamment l'invisibilité de ces problématiques dans le milieu de l'enseignement, et s'interroge sur l'effet de ce dernier sur l'usure observée chez cette catégorie de travailleuses. Toutes ces problématiques se trouvent au cœur de la décision de maintien ou de renoncement à l'emploi chez les enseignantes de maternelle. Pour CauBareille, ce questionnement s'avère essentiel au « sentiment d'efficacité personnelle » développé par les travailleuses en fin de carrière, en regard des transformations de leur milieu de travail et de leurs propres transformations avec l'âge.

Pour leur part, Nuno Bessa et Marianne Lacomblez questionnent les normes et les valeurs dans l'aménagement du travail posté, en prenant comme point d'ancrage le travail nocturne et le travail par roulement, ainsi que leurs effets pathogènes sur la santé des travailleurs. La recension des travaux scientifiques sur la santé et l'organisation du travail confirment, pour le moins, l'impact nocif de ces types de travail sur les travailleurs, notamment pour les plus âgés d'entre eux. De plus, l'organisation du temps de travail pour ces types de poste n'est pas exempte de contraintes juridiques et légales, ce qui influence de manière substantielle les pratiques des entreprises. À travers le récit d'une intervention, Nusso et Lacomblez ont cherché à comprendre « comment concilier les évolu- tions du temps de travail avec les effets du travail sur la santé des travailleurs ? » C'est dans le modèle tripolaire de l'ergologie que les deux chercheurs ont analysé les résultats. En effet, selon ce modèle, I'activité du travail est au centre d'un espace de tension entre deux pôles : le pôle des marchés (valeurs marchandes et quantifiables) et le pôle du politique (la recherche du bien commun, le rôle du droit du travail). Le pôle de l'activité est donc l'espace dans lequel les acteurs organisent et structurent les normes en fonction de la variabilité des situations auxquelles ils sont confrontés.

Alexandre Morais, quant à lui, examine les actions conduites en ergonomie dans le Groupe PSA eu égard à I'application de I'accord senior signé par l'entreprise. II est nécessaire de rappeler que, dans le but d'améliorer le taux d'emploi des seniors, le gouvernement français a obligé les entreprises de plus de 50 salariés à adopter un accord favorisant I'emploi des seniors, entente qui comporte six grands domaines d'action visant à améliorer l'employabilité de cette catégorie de travailleurs. Après avoir présenté les grandes lignes de cette entente, l'auteur s'intéresse plus particulièrement aux articles impliquant l'ergonomie (adaptation des conditions de travail, aménagement des fins de carrière, transition entre activité et retraite, etc.) ainsi qu'à leur mise en œuvre dans le Groupe PSA. Morais distingue deux types de traitement : curatif, à travers I'amélioration de l'employabilité des salariés présentant des restrictions nécessitant l'aménagement d'un poste; et, préventif, à travers l'ergonomie. Ce second traitement vise à développer des processus à long terme en vue d'adapter les conditions de travail tout au long de la vie professionnelle. L'auteur souligne également l'importance de la communication, de la sensibilisation et de la formation comme outils indispensables à I'activité des ergonomes au sein du groupe. Il conclut que si le traitement curatif semble 
maîtrisé par le Groupe, l'approche préventive risque de prendre davantage de temps à être mise en place.

La contribution de Véronique Poète porte également sur les accords seniors, avec un intérêt plus marqué pour la question de la pénibilité. En effet, I'auteure s'indigne du foisonnement des différents décrets, accords et autres demandes visant à réduire la pénibilité dans les entreprises, sans véritablement parvenir à une solution concertée qui s'inscrit dans le temps. Pour cela, elle cite deux exemples d'entreprises dont les négociations sur ce sujet ont dévié vers des problématiques beaucoup plus ciblées et plus locales, alors que c'est vers une politique de prévention générale que devrait s'inscrire la problématique de la pénibilité. Pour Poète, les causes de ces déviations sont d'abord dues à un manque de consensus sur la signification du terme " pénibilité » se limitant, dans la plupart des cas, à une vision étroite d'une spécificité pénible du travail, et non à l'ensemble des facteurs pouvant causer cette pénibilité. L'auteure constate également une déconnexion entre les différents acteurs quand il s'agit de la mise en commun des apprentissages retenus des différents accords sur la pénibilité. Par ailleurs, Poète critique la méthode d'intervention des ergonomes dans ce genre de situation et elle propose un " décloisonnement de la démarche » afin de donner une vision plus claire et entière du travail ainsi que de ses conditions internes (milieu du travail) et externes (expérience du travail).

En dernier lieu, Nadia Heddad, Sylvain Biquand et Sabine Guérin décrivent une intervention ergonomique menée auprès de gardiens d'immeuble travaillant dans la régie immobilière de la ville de Paris. En effet, les auteurs ont saisi l'opportunité du plan senior et d'un appel d'offres lancé par l'entreprise en question pour faire une intervention. Celle-ci consiste à prendre en considération le travail du gardien dans sa globalité afin de créer un outil à partir d'indicateurs observés lors de l'activité des travailleurs. Cet outil s'est construit sur une double évaluation : I'une, à l'usage de I'inspecteur en charge de l'appréciation du travail; I'autre, à l'usage du gardien. Ceci permet de réunir des éléments objectivables et subjectifs qui amènent à saisir la complexité des situations de travail dans son entièreté. Les auteurs pensent que l'approche ainsi développée devrait permettre de tenir compte des parcours professionnels et, ainsi, de passer d'un management de contrôle vers un management d'aide à la réalisation du travail.

Pour résumer, cet ouvrage reprend les grands thèmes classiques de la relation " âge-travail » selon une perspective ergonomique. Les contributions sont assez courtes et concises, ce qui permet au lecteur de se faire une idée globale de la question. Cela dit, il nous semble que I'analyse critique des différentes postures théoriques jalonnant ces thèmes n'est pas assez présente. L'ouvrage apporte, certes, quelques exemples concrets d'intervention sur le terrain, mais nous ne pouvons pas dire qu'il constitue un matériau empirique substantiel qui permettrait de faire le tour de la question posée. Par ailleurs, la présence d'une ligne directrice aurait facilité la lecture et l'appréciation des contributions des différents auteurs. Soulignons que certaines contributions atteignent un degré assez élevé de spécialisation, ce qui rend leur compréhension difficile pour un public non averti. En somme, il s'agit d'un ouvrage réalisé par des ergonomes qui s'adressent surtout aux ergonomes, car ses apports sont souvent liés à la posture et à la démarche que devrait adopter l'ergonomie contemporaine dans le traitement de la question de l'âge au travail.

\section{Siham Abouaissa \\ Chargée de cours au Département des relations industrielles \\ Université Laval}

